\title{
Phylogenetic analysis of H1N1 sequences from pandemic infections during 2009 in India
}

\author{
Guntupally Balaswamy Arti Flavia \& Kalimuthusamy Natarajaseenivasan*
}

Division of Medical Microbiology, Department of Microbiology, School of Life Sciences, Bharathidasan University, Tiruchirappalli - 620 024, Tamilnadu, India; Kalimuthusamy Natarajaseenivasan - Email: natarajaseenivasan@rediffmail.com; Phone: +914312407082; Fax: +914312407045; *Corresponding author

\begin{abstract}
:
Since April 2009, a serious pandemic infection has been rapidly spread across the world. These infections are caused due to the novel swine origin influenza A (H1N1) virus and hence these are commonly called as "Swine Flu”. This new virus is the reassortment of avian, human and swine influenza viruses and thus it has a unique genome composition. There are 16 different types of hemagglutinin (HA) and 9 different types of neuraminidase (NA) that can be genetically and antigenetically differentiated. The first influenza A virus isolated from pigs was of the H1N1 subtype and these viruses have been reported to cause infection in pigs in many countries. The outbreak of this virus has been transmitted from pigs to humans. This new reassorted (exchange of genes) virus which is the cause of 2009 pandemic infections has the ability to spread from human to human. This spread of infection should be brought to an end. In this study, a phylogenetic analysis of the nucleotide sequences of the RNA segments of human H1N1 viruses was carried using MEGA version 4.0 to demonstrate the route map of infection to India. Phylogenetic analysis of the sequences from India, published in Influenza Virus Resource (a database that integrates information gathered from the Influenza Genome Sequencing Project of the National Institute of Allergy and Infectious diseases (NIAID) and the genbank of the (NCBI)) was retrieved and used for the analysis. The results showed that the various segments of the Indian isolates clustered well with the sequences from American, Asian and European countries and thus indicating the transmission of viruses from these places to India.
\end{abstract}

Keywords: H1N1 virus, Phylogenetic analysis, India, Swine Flu

\section{Background:}

The 1918 influenza pandemic (also called as "mother of all pandemics") infected more than one quarter of the world's population and claimed about 20 to 50 million human lives. The influenza virus slowly turns out to be a zoonotic threat and there are 2 main reasons for it. One reason is high error rate during genomic replication. The other reason is the segmented genome, which facilitates reassortment between different viral strains that infect the same cell [1]. Influenza virus can spread through air and this enables the occurrence of pandemic infections.

Swine Flu is a respiratory disease caused by a new strain of the Influenza virus type $\mathrm{A}$. The strain is known as $\mathrm{H} 1 \mathrm{~N} 1$ and is similar to the one found in seasonal flu outbreaks, except that it contains a reassorted genetic material of birds, humans and pigs. The genetic material of Influenza virus contains eight segments of single stranded RNA. Of its 8 segments of RNAs, 2 polymerase genes, PB2 and PA, were from the avian virus of North American lineage and were introduced into swine populations around 1998. The other polymerase gene, PB1, also evolved recently from a human seasonal influenza (H3N2) virus around the same year. Hemagglutinin (HA), nucleoprotein (NP) and nonstructural (NS) protein genes descended directly from the classic swine influenza A virus of North American lineage, which can be traced back to the 1918 virus. Originating from the Eurasian Swine virus, the remaining 2 genes, neuraminidase (NA) and matrix(M), were introduced from birds around 1979 [2].In California, 2 viral isolates which were genetically similar and was found to contain a novel genetic combination of segments from previous swine influenza viruses that have circulated in the United States since 1999, genes from swine viruses of the Eurasian Lineage and genes from avian influenza viruses were isolated from children suffering from respiratory illness.
Neither of these children had exposure to swine or to each other, indicating that this virus was capable of Human to Human transmission [3].

In this regard such a type of H1N1 virus first detected in Mexico in April 2009 has subsequently spread to other countries. This was originally referred to as "swine flu" because laboratory testing showed that many of the genes in this new virus were very similar to those viruses infecting pigs in North America. It became apparent in the early days of the outbreak that the virus can be directly transmitted between humans.

In India, swine flu started around May 2009 from international travelers. India's first swine flu death was on August $4^{\text {th }}$ of a girl in Pune. Since then it has taken out lot of lives throughout the country. Travel in and out of India has remained unaffected at large. Within India travel to Maharashtra and Pune city in particular was affected. Though various measures have been implemented against the spread of the virus, finding out the genomic sequences of different viral isolates and their lineage of origin play a major role in total eradication of the illness. Thus in the present study, a phylogenetic analysis of the nucleotide sequences of the RNA segments of human H1N1 viruses was carried to demonstrate the route map of infection to India.

\section{Methodology:}

Phylogenetic analysis of all eight RNA segments of H1N1 was carried out. Nucleotide sequences were downloaded from Influenza Virus Resource, a database that integrates information gathered from the Influenza Genome Sequencing project of the National Institute of Allergy and Infectious diseases (NIAID) and the Genbank of the NCBI. Only the sequences of the strains isolated from Homo sapiens were taken into consideration. NCBI 
Blast was used to identify the sequence identity. Nearly 500 sequences of each segment were used to analyze the 8 segments. These include the nucleotide sequences of polymerases PB1, PB2; hemagglutinin; neuraminidase; matrix proteins; nonstructural proteins. The selected sequences were aligned using alignment explorer in Mega 4.0 with default parameters. Phylogenetic and molecular evolutionary analyses were conducted using MEGA version 4. Phylogenetic tree was built by neighbor joining method. The total numbers of sequences analyzed were more than 100.

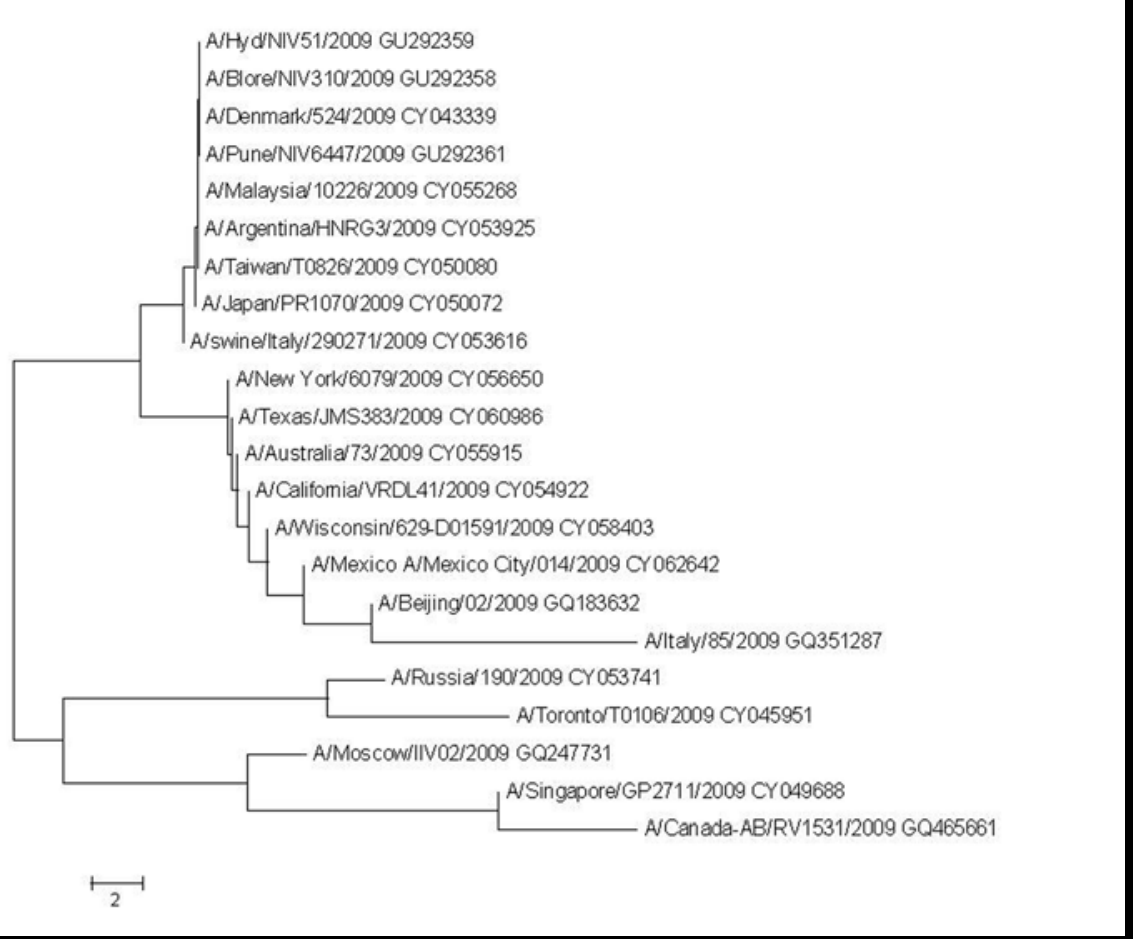

Figure 1: NJ plot was made for all the 8 segments of selected influenza viruses using boot strap analyses at 500 replicates. (1) Polymerase PB2

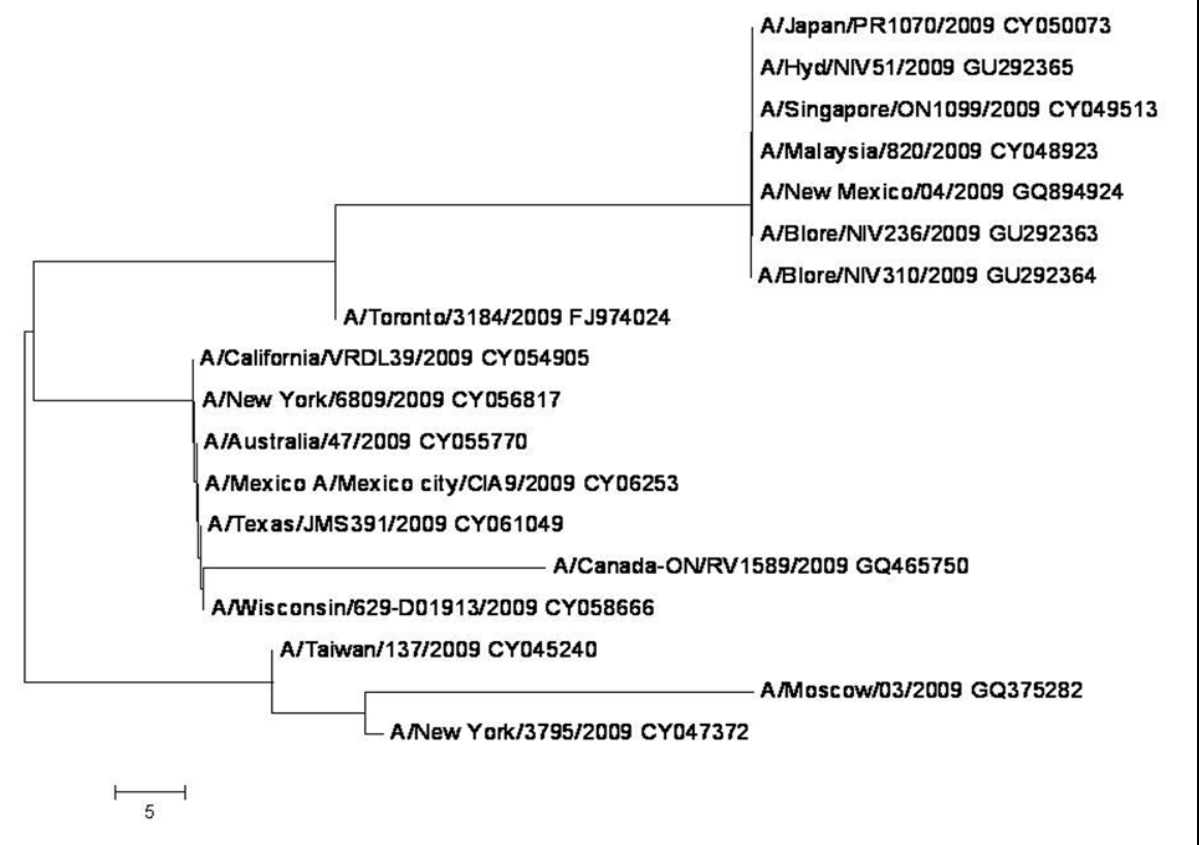

Figure 2: NJ plot was made for all the 8 segments of selected influenza viruses using boot strap analyses at 500 replicates. (2) Polymerase PB1 


\section{Bioinformation}

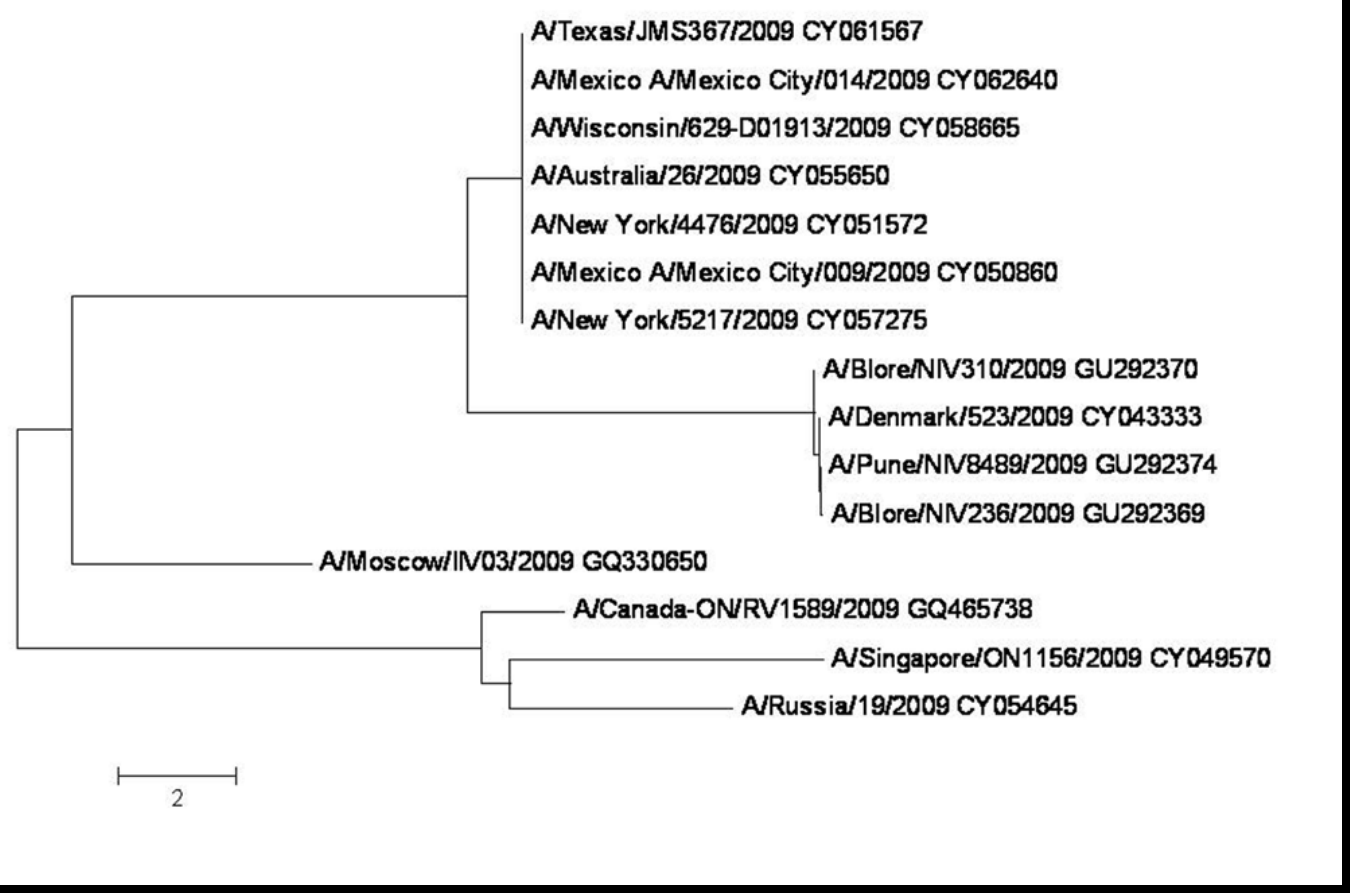

Figure 3: NJ plot was made for all the 8 segments of selected influenza viruses using boot strap analyses at 500 replicates. (3) Polymerase PA

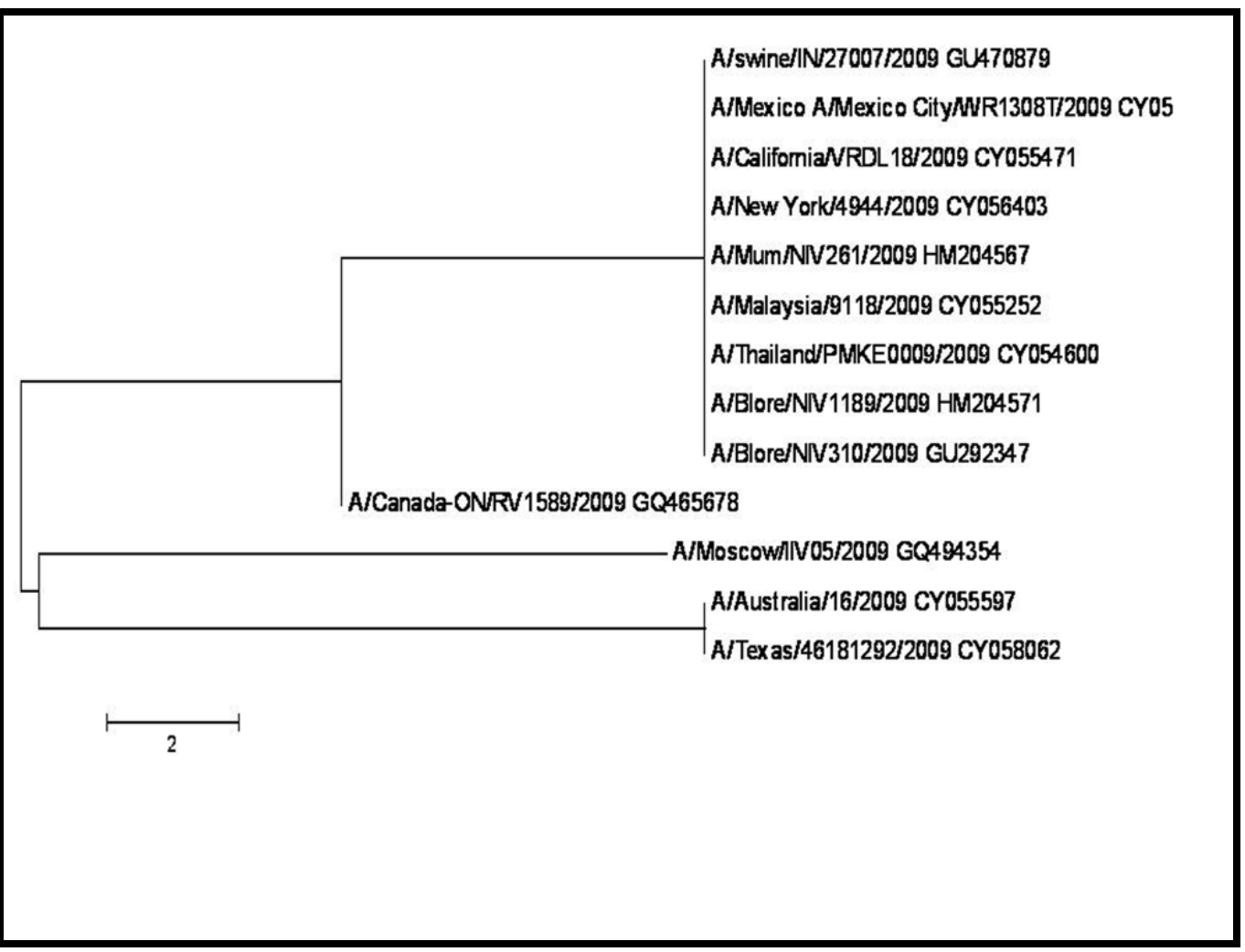

Figure 4: NJ plot was made for all the 8 segments of selected influenza viruses using boot strap analyses at 500 replicates. (4) Hemagglutinin 


\section{Bioinformation Volume 5}

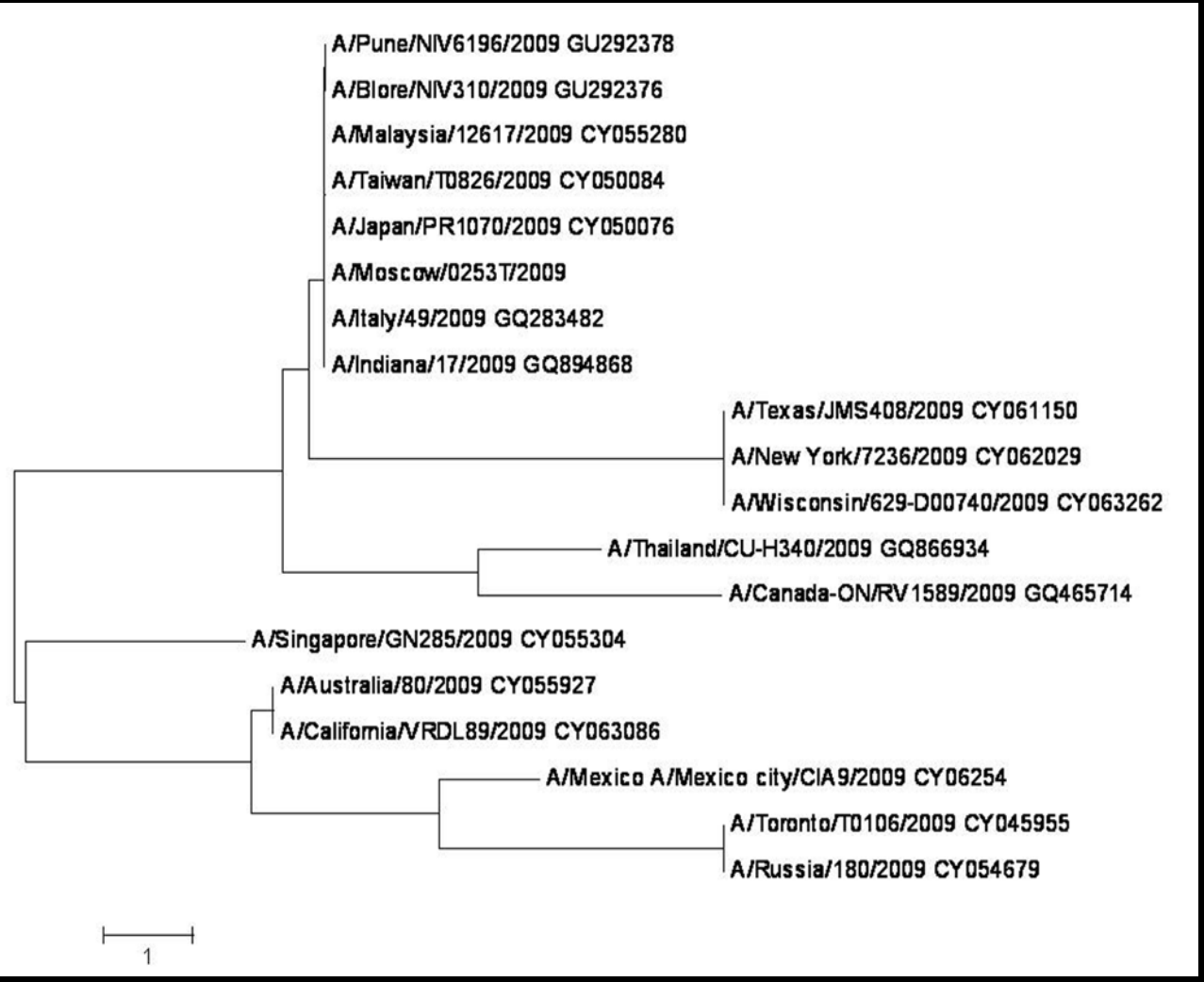

Figure 5: NJ plot was made for all the 8 segments of selected influenza viruses using boot strap analyses at 500 replicates. (5) Nucleocapsid Protein,

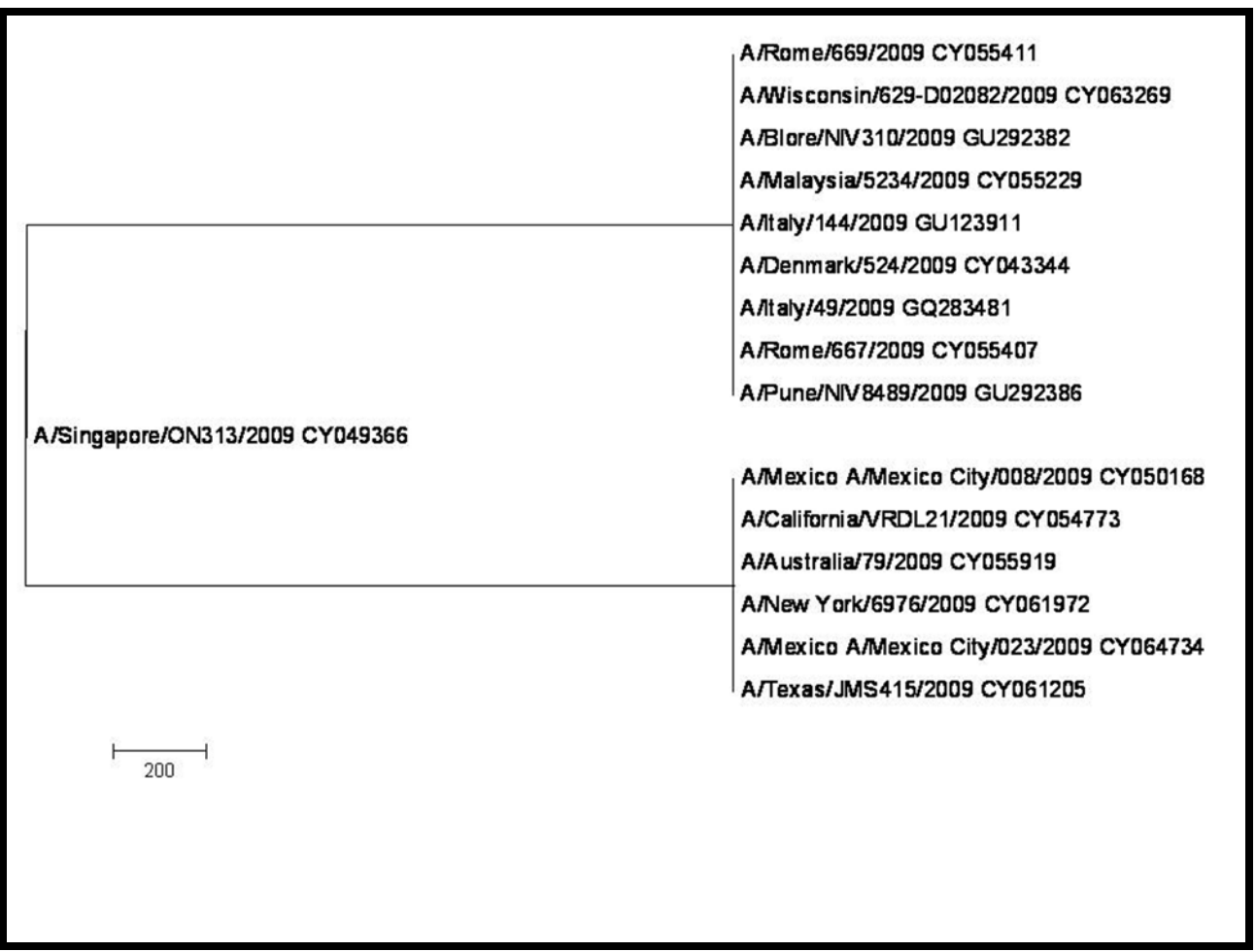

Figure 6: NJ plot was made for all the 8 segments of selected influenza viruses using boot strap analyses at 500 replicates. (6) Neuraminidase 


\section{Bioinformation Volume 5}

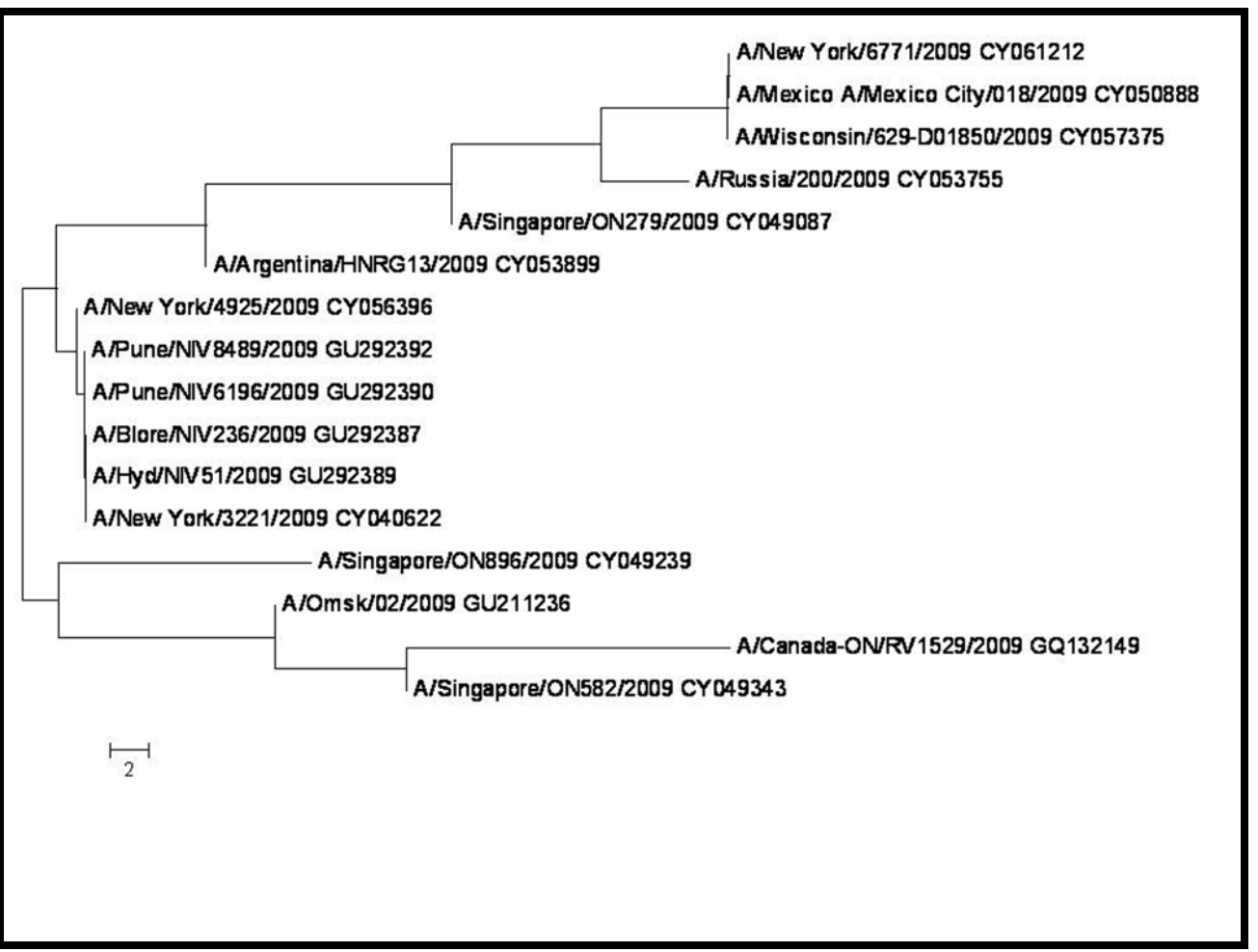

Figure 7: NJ plot was made for all the 8 segments of selected influenza viruses using boot strap analyses at 500 replicates. (7) Matrix protein

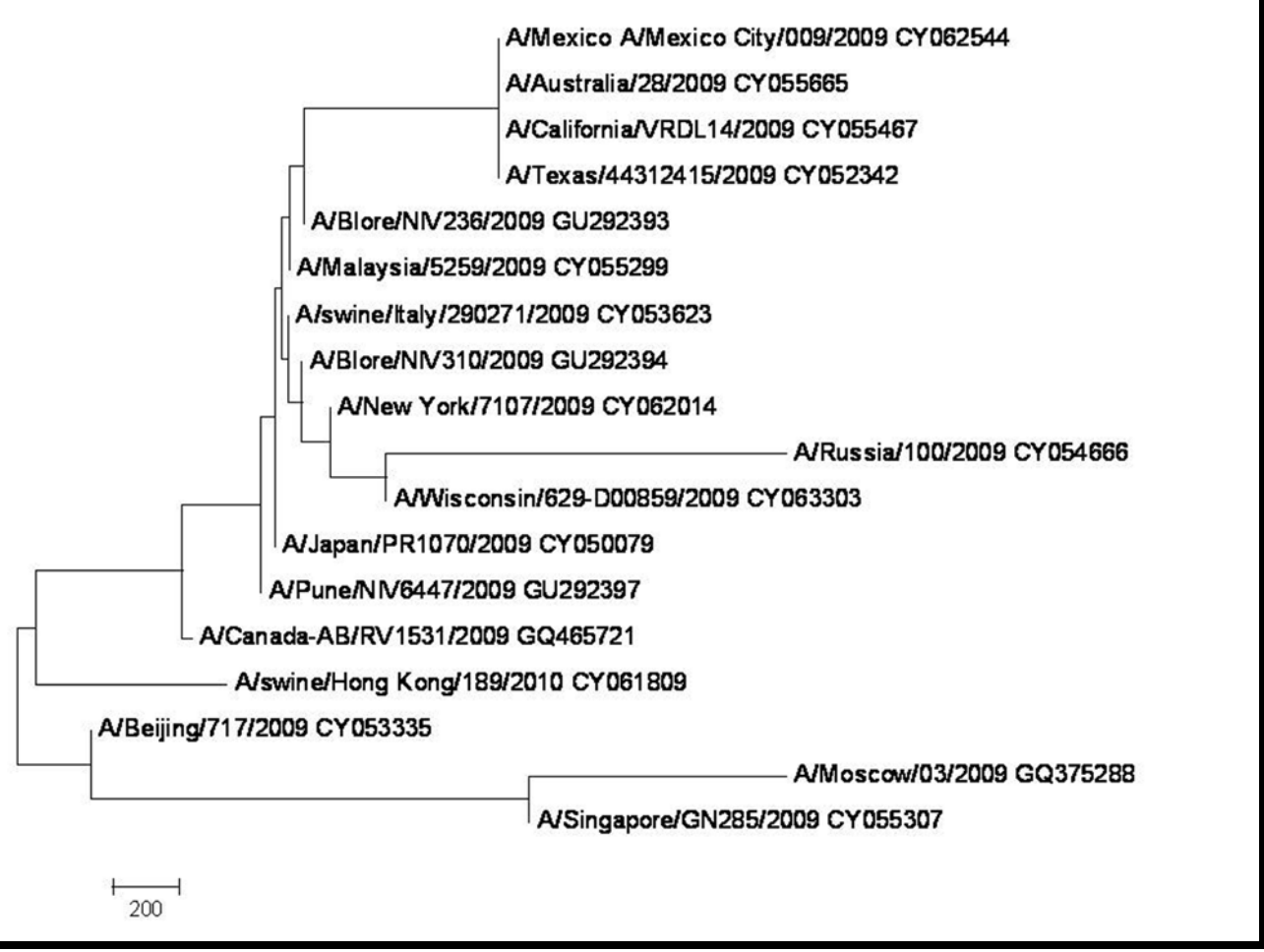

Figure 8: NJ plot was made for all the 8 segments of selected influenza viruses using boot strap analyses at 500 replicates. (8) nuclear export protein. 


\section{Discussion:}

Pigs in North America are been suspected as the "mixing vessel" and these pigs thus play a significant role in interspecies transmission of influenza viruses [4]. A novel 2009 human virus, circulating in USA, Europe and Asia is known to be a reassorted virus and our studies show that it is these viruses that have spread rapidly across worldwide leading to pandemic infections.

Nucleotide sequences of all the 8 segments encoded by the new influenza A (H1N1) virus which was pandemic in 2009 were used. Sequences from India (especially Bangalore, Hyderabad and Pune) were retrieved from the Influenza Virus Resource. Similarity search was performed between these sequences and the other sequences from other countries retrieved from the Influenza Virus resource database using Blastn. The best matches were selected and phylogenetic analysis were done using MEGA version 4.

Phylogenetic analysis showed that the sequences from Indian isolates clustered well with Asian and American countries. The virus is named based on the type of surface proteins i.e., Hemagglutinin (segment 4) and Neuraminidase (segment 6). Hence analyses of these two proteins are more important. The analysis done by Danishuddin et al. (2009) showed that the hemagglutinin sequences of two strains from USA and one strain from Asia from the 2009 outbreaks clustered with the swine influenza sequences from H1N2 subtype recovered from 1999 - 2004 outbreaks in USA and the segment coding for neuraminidase gene (NA) of new human virus clustered with the swine influenza viruses from Asia and Europe from 1992 - 2007 outbreaks. Our analysis also showed that hemagglutinin sequences from Indian isolates clustered with New York and Mexico (Figure 4) and the segment coding neuraminidase clustered with Italy and Rome i.e., European countries (Figure 6). All these sequences were of Swine lineage. Based on the analysis done by Gorman [5] NP nucleotide sequences showed that human and classical swine viruses share a single common ancestor and it is estimated to have emerged in 1912 to 1913. Our analysis showed that the segment 5 nucleocapsidproteins clustered with the Asian countries Singapore, Malaysia, Japan and Taiwan (Figure 5) Segment 1 and 3 the polymerase genes clustered well with the European country Denmark (Figure 1 \& 3) while the segment 2 and 7 polymerases i.e., the Matrix proteins M1 and M2 clustered with American countries (New York and New Mexico) (Figure 2 \& 7). The other sequences clustered with Asian countries such as Malaysia, Singapore, and Japan. On analyzing the sequences from Asian countries they were highly similar to the sequences of Indian isolates and they were found to have the gene segments of the virus originated in Swine of North America (Figure 8). Travelers to India who are infected or carriers of swine flu from other countries, due to change in climatic conditions and other such factors could have been the reason for spread of infections to India.

\section{Conclusion:}

More accurate rates of evolution and dates of common ancestors can be obtained from nucleotide phylogenies. This is because protein evolution is dependent on nucleotide evolution and nucleotide sequences contain much more information [6]. According to Larry Brilliant, an epidemiologist, we are entering into "age of pandemics" wherein outbreaks of infectious diseases will become increasingly common. Zoonotic diseases are rising rapidly and this is because the habitats of the animals are being destroyed and they begin to live more closely than ever before. As a result, we share our viruses. The ability of the viruses to reassort their genes is the main reason for the spread of A (H1N1) influenza virus such that the organism was viable in changing climatic conditions. Influenza virus can also spread through air and this has also enabled the spread of the infection easily around the world.

\section{Acknowledgment:}

This work was supported by the Indian Council of Medical Research (ICMR) Grants 5/13/88/06/NCD-III. We thank the Vice-Chancellor, Bharathidasan University for the facilities provided.

\section{References:}

[1] M Danishuddin et al. Bioinformation (2009) 4: 94 [PMID: 20198180]

[2] GW Chen \& S Ru, Emerging Infectious Diseases (2009) 15: 12 [PMID: 19998748]

[3] SJ Sullivan et al. Mayo Clin Proc. (2010) 85: 64 [PMID: 20007905]

[4] GM Nava et al. Euro Surveill. (2009) 14: 19228 [PMID: 19497253]

[5] OT Gorman et al. Journal of Virology (1991) 65(7): 3704 [PMID: 2041090]

[6] SM Stroh et al. Biology Direct (2009) 4:18 [PMID: 19457254]

Edited by $P$ Kangueane

Citation: Flavia \& Natarajaseenivasan, Bioinformation 5(10): 416-421 (2011) License statement: This is an open-access article, which permits unrestricted use, distribution, and reproduction in any medium, for non-commercial purposes, provided the original author and source are credited. 